\title{
Through-Mask Electrochemical Micromachining of Aluminum in Phosphoric Acid
}

\author{
T. Baldhoff, ${ }^{\mathrm{a}, \mathrm{z}}$ V. Nock, ${ }^{\mathrm{b}}$ and A. T. Marshall ${ }^{\mathrm{a}}$ \\ ${ }^{a}$ Department of Chemical and Process Engineering, University of Canterbury, Christchurch, New Zealand \\ ${ }^{b}$ Department of Electrical and Computer Engineering, University of Canterbury, Christchurch, New Zealand
}

\begin{abstract}
Aluminum micro-channels have been machined in phosphoric acid via mass-transfer limited electrochemical dissolution through photoresist masks. The results of shape evolution experiments using a rotating disk electrode are presented in terms of the dimensions, shape profile and uniformity of the machined micro-channels. The influences of applied potential, cumulative passed charge and hydrodynamic conditions on the shape evolution process are discussed. Experimental results are compared with a shape evolution model assuming the rate of aluminum removal is solely controlled by diffusive mass transfer. The degree of agreement between experimental and simulated results depends mainly on the hydrodynamic conditions in the electrochemical cell and indicates a shift from purely diffusive to mixed convective-diffusive mass transfer. The feasibility of electrochemical aluminum micromachining is demonstrated by fabricating microfluidic test structures with well-defined geometries and smooth surfaces.

(C) The Author(s) 2017. Published by ECS. This is an open access article distributed under the terms of the Creative Commons Attribution 4.0 License (CC BY, http://creativecommons.org/licenses/by/4.0/), which permits unrestricted reuse of the work in any medium, provided the original work is properly cited. [DOI: 10.1149/2.0441709jes] All rights reserved.

(cc) BY
\end{abstract}

Manuscript received May 3, 2017. Published June 28, 2017.

Through-Mask Electrochemical Micromachining (TMEMM) is an unconventional machining process, in which a metal substrate is made the anode in an electrochemical cell and thereby dissolved. ${ }^{1,2}$ Localized material removal is achieved by covering the substrate with an insulating mask. The use of common photolithography processes for the creation of the mask enables the fabrication of micrometer-sized structures. Thereby, TMEMM can be used in two ways to fabricate microfluidic devices: firstly, cavities and channels can be machined into the substrate surface and secondly, through-holes and slits can be machined into metal foils or shims. ${ }^{1}$ Compared to classical wet chemical and dry etching processes, TMEMM offers higher rates of material removal, better control of surface finish, more selective machining of substrates and enhanced process safety. ${ }^{3-5}$ In addition, combining photolithography with electrochemical dissolution processes is expected to enable the fabrication of a large number of microfluidic devices in parallel and at low cost. ${ }^{6}$

Aluminum is widely used as a construction material for the manufacture of microfluidic devices such as micro-heat exchangers, ${ }^{7}$ microreactors $^{8}$ and micro-fuel cells. ${ }^{9}$ Other areas of interest are the fabrication of intricate parts in optical, electronic, automotive and aerospace systems. ${ }^{10,11}$ Its chief advantages compared to other materials used in these fields are its high thermal conductivity, low electrical resistivity, easy machinability and low density. In addition, it is possible to modify the surface structure of aluminum substrates via the formation of highly ordered, porous oxide films along the surface. ${ }^{12,13}$ It has been demonstrated, that these films can be subsequently used to incorporate noble metal catalysts along the surface of micro-channels within a micro-reactor. ${ }^{14-16}$

Electrochemical dissolution of aluminum in phosphoric acid is known to produce reflective aluminum surfaces with sub-micrometer surface roughness at sufficiently high electrolyte concentration and temperature. ${ }^{17}$ This property makes it attractive for TMEMM of micrometer-sized structures made from aluminum. The observed leveling and brightening effect is caused by the mass transfer limitation of the dissolution process ${ }^{18}$ and is frequently linked to the formation of oxide or salt films along the surface. ${ }^{19}$ Which type of mass transfer dominates the dissolution process - whether it is diffusion, natural or forced convection - thus has a marked influence on the resulting shape profiles ${ }^{20-26}$ and depends on the hydrodynamic conditions in the electrochemical cell.

The use of TMEMM of aluminum has thus far been demonstrated for the fabrication of micrometer-sized cantilevers, ${ }^{27}$ channels, ${ }^{28}$ lenses $^{29}$ and pits. ${ }^{30}$ The majority of this work ${ }^{27-29}$ was conducted in mixtures of perchloric acid, acetic acid and ethanol, which are linked

${ }^{\mathrm{z}}$ E-mail: tobias.baldhoff@pg.canterbury.ac.nz to specific safety concerns due to their reactivity, flammability and limited long-term stability. In addition, previous studies focused mainly on device fabrication and the influence of current density, electrolyte composition, machining time or initial surface structure. At present few numerical or experimental studies of TMEMM exist with a focus on the influence of mass transfer conditions on the resulting depth, width, shape profile and uniformity of machined micro-channels. Rosset and Landolt ${ }^{31}$ and Rosset et al. ${ }^{32}$ presented results on shape evolution during TMEMM of stainless steels using spray-etching equipment and a flow channel cell, respectively. Winkelmann and Lang ${ }^{33}$ studied the effect conductivity variations on shape evolution of a steel anode using a flow channel cell for a modified TMEMM process employing a masked cathode tool. This work is complemented by investigations of chemical etching. Shin and Economou ${ }^{22-24}$ were the first to numerically study the influence of natural and forced convection in the vicinity of a mask opening during mass-transfer limited, chemical etching on shape evolution. More recently, other studies ${ }^{25,34-36}$ have employed different numerical methods to solve similar problems.

The aim of this study is to investigate the effect of mass transfer, namely the change from diffusion to mixed convection-diffusion control, on the shape evolution during TMEMM of aluminum in phosphoric acid. To this end shape evolution experiments were conducted using a Rotating Disk Electrode (RDE), which allowed experiments to be executed under controlled hydrodynamic conditions. From the analysis of electrochemical impedance spectra ${ }^{37}$ it is known that during aluminum dissolution in phosphoric acid the surface is covered with a thin alumina film at all times. As the film's thickness lies on the order of nanometers, its presence can be neglected for the study of shape evolution during TMEMM. The experimental results were thus compared with a shape evolution model previously developed by Madore et al. ${ }^{38}$ for TMEMM of titanium, assuming that shape changes are determined by diffusive mass transfer only. The model was solved numerically using both a Boundary Element Method (BEM) and a Finite Element Method (FEM). The influence of applied potential on the dimensions and uniformity of machined channels was also studied. The feasibility of TMEMM for the fabrication of microfluidic devices made from aluminum was demonstrated by machining several test structures.

\section{Experimental}

All experiments were performed with a recessed RDE made from commercially pure aluminum $(\mathrm{Al} \geq 99.00 \%, \mathrm{Si}+\mathrm{Fe}<1.00 \%, \mathrm{Cu}<$ $0.05 \%, \mathrm{Mn}<0.05 \%, \mathrm{Zn}<0.10 \%$, Ti $<0.05 \%$, Wakefield Metals). Disks were machined from $2 \mathrm{~mm}$ thick sheet material to a diameter of $15 \mathrm{~mm}$. Prior to each experiment the disks were mechanically polished with silicon carbide paper of different grit sizes down to P2000 and subsequently brought to a mirror finish by polishing with 
(a)

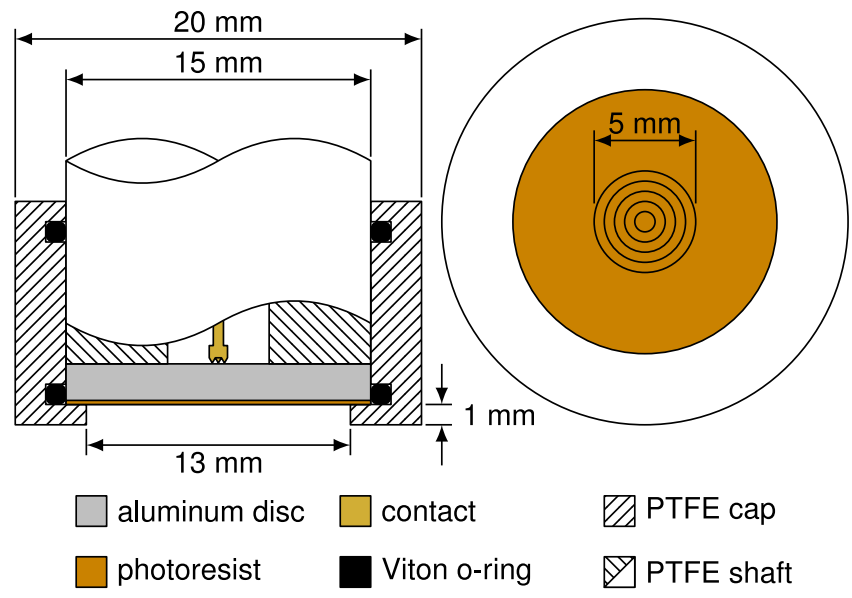

Figure 1. (a) Cross-sectional and (b) top view of the recessed RDE showing the test pattern to be machined consisting of five concentric rings.

alumina slurry of $1 \mu \mathrm{m}$ and $0.3 \mu \mathrm{m}$ particle size for one hour each on velvet cloth (ProSciTech) using a LaboPol-2 polishing machine with a LaboForce-1 sample mover (Struers). Afterwards, grease and particles were removed via ultrasonication in acetone, methanol and isopropanol (analytical reagent grade, Thermo Fisher Scientific) for 5 minutes each and the disks were dried with pressurized nitrogen. The polished disks were coated with positive-tone AZ1518 photoresist (Microchem) with an average thickness of $1.95 \mu \mathrm{m}$ using a spincoater (PWM32, Headway Research) and subsequently pre-baked for $90 \mathrm{~s}$ on a hotplate at $100^{\circ} \mathrm{C}$. The photoresist film was exposed to UV light on a Karl-Suss MA6 mask aligner via proximity exposure and then developed in AZ MIF 326 (Microchem) for $30 \mathrm{~s}$. Afterwards the samples were post-baked for $120 \mathrm{~s}$ on a hotplate at $120^{\circ} \mathrm{C}$ to harden the photoresist mask. The disks were inserted into a PTFE cap creating a $1 \mathrm{~mm}$ deep and $13 \mathrm{~mm}$ wide recess (Figure 1) and then mounted on a AFE6MB RDE shaft, whose rotation rate was controlled with a Modulated Speed Rotator (Pine Research Instrumentation).

Electrochemical experiments were controlled with a Reference 3000 potentiostat (Gamry Instruments) in a custom-made $100 \mathrm{~mL}$ glass cell. A $4 \mathrm{~cm}^{2}$ platinum foil connected to a platinum wire served as the counter electrode. All potentials are reported against a $\mathrm{Ag} \mid \mathrm{AgCl}$ electrode $\left(c_{\mathrm{KCl}}=4.77 \mathrm{~mol} / \mathrm{kg}, E_{\mathrm{Ag} \mid \mathrm{AgCl}}\left(75^{\circ} \mathrm{C}\right)=0.153 \mathrm{~V}\right.$ vs. SHE$)$. The reference electrode was connected to a Luggin capillary with an outer diameter of $2 \mathrm{~mm}$ and held concentrically underneath the RDE at a distance of $10 \mathrm{~mm}$. Before each experiment the glass cell was ultrasonically cleaned in a mixture of isopropanol and deionized water $(18.2 \mathrm{M} \Omega \mathrm{cm}$, arium 611UV system, Sartorius Stedim Biotech), rinsed several times with deionized water and dried in an oven at $105^{\circ} \mathrm{C}$. The glass cell was immersed in a water bath, whose temperature was thermostat-controlled at $75^{\circ} \mathrm{C}$ within $\pm 1^{\circ} \mathrm{C}$ via a copper coil connected to a GD120 thermostat and a LTC1 circulation bath (Grant Instruments). The electrolyte was made from $85 \%$ phosphoric acid (ACS grade, Thermo Fisher Scientific) and used as received.

For shape evolution experiments a mask pattern consisting of five concentric rings with center line radii of $0.5,1.0,1.5,2.0$ and $2.5 \mathrm{~mm}$ was used (Figure 1). This pattern was chosen to limit the influence of convective mass transfer to the radial flow component along the disk surface only and to minimize the influence of neighboring mask openings on the shape evolution process of a particular ring. On average the difference between the inner and the outer radius of each annular mask opening amounted to $11.5 \mu \mathrm{m}$ resulting in a mask opening aspect ratio of 0.34 and an initially exposed aluminum surface of $0.542 \mathrm{~mm}^{2}$. Prior to the shape evolution experiments it was tested, whether the recess created by the PTFE cap (Figure 1) and the cap itself impacted the hydrodynamic conditions in the vicinity of the aluminum disk surface. To this end the uniform accessibility to mass transfer and the absence of edge effects were confirmed by determining the diffusion coefficient of $\mathrm{Fe}^{2+}$ and $\mathrm{Fe}^{3+}$ on a gold RDE (AFE2M050AU, Pine Research Instrumentation) with an active area $5 \mathrm{~mm}$ in diameter in $0.5 \mathrm{M} \mathrm{H}_{2} \mathrm{SO}_{4}$ with and without a recess. No influence of the recess or the cap was detected and the results were found in good agreement with literature values.

Current transients of electrochemical aluminum dissolution through this mask pattern were recorded at an applied potential of $1.4 \mathrm{~V}$ in a quiescent solution and using different rotation rates of 100 , 400 and $900 \mathrm{rpm}$ as well as at $5.4 \mathrm{~V}$ and $100 \mathrm{rpm}$. The amount of removed material was governed by adjusting the cumulative passed charge during an experiment to $0.2,0.5,1.0$ or $2.0 \mathrm{C}$. All experiments were conducted at least in duplicate. Afterwards, the photoresist was stripped and the disks were cleaned by rinsing them with isopropanol, methanol and acetone. Subsequently, the samples were examined via light microscopy (Olympus BX60, Leica DFC320), SEM (JEOL JSM 7000F) and optical profilometry (Bruker ContourGT). Cross-sections for further microscope examination were obtained by encasing samples in epoxy, sectioning them and mechanically polishing the cross-sectioned surface to a mirror finish with alumina slurry. For SEM imaging cross-sectioned samples were also coated with a thin chromium film via sputtering (EMS150T ES) to reduce charging at the edge between the aluminum and the epoxy.

\section{Simulation}

Mathematical problem.-The formulation of the mathematical problem describing the shape evolution during TMEMM has been presented in detail by West et al. ${ }^{39}$ and Madore et al. ${ }^{38}$ In the case of mass-transfer limited electrochemical dissolution, a tertiary current distribution can be presumed to govern the local etch rate along the metal surface, hence the influence of ohmic resistances and electrode kinetics can be neglected. In the absence of convective mass transfer and using a pseudo-steady state assumption for the relaxation of the concentration field $(c)$, the convection-diffusion equation simplifies to yield the Laplace equation:

$$
\nabla^{2} c=0
$$

Assuming $100 \%$ current efficiency, the rate of displacement (v) of any point along the metal is related to the current density normal to the surface $\left(i_{n}\right)$ via Faraday's law:

$$
\mathbf{v}=\frac{M}{z F \rho} i_{\mathrm{n}} \mathbf{n}
$$

Herein, $M$ denotes the molar mass of the metal, $z$ the charge transfer number, $F$ the Faraday constant, $\rho$ the metal density and $\mathbf{n}$ the unit normal vector. The current density distribution along the metal is in turn linked to the gradient of the concentration field close to the surface via Fick's law, where $D$ is the diffusion coefficient:

$$
i_{\mathrm{n}}=z F D \nabla c
$$

The following boundary conditions apply in order to solve the Laplace equation along the boundary:

$$
\begin{gathered}
\partial c / \partial n=0 \text { (along the insulator and symmetry line) } \\
c=c_{\mathrm{s}} \text { (along the metal surface) }
\end{gathered}
$$$$
c=0 \text { (along the diffusion layer boundary) }
$$

The concentration gradient normal to the symmetry line and to the insulator surface is assumed to be zero, as there is no flux across those boundaries. The concentration along the metal surface is assumed to be constant corresponding to the presence of a surface film. The concentration along the boundary of the diffusion layer equals the bulk concentration.

For simplicity we assume that the curvature of each ring is negligible due to its small width and large radius. Thus, the initial geometry of the problem corresponds to an isolated, infinitely long line electrode, 


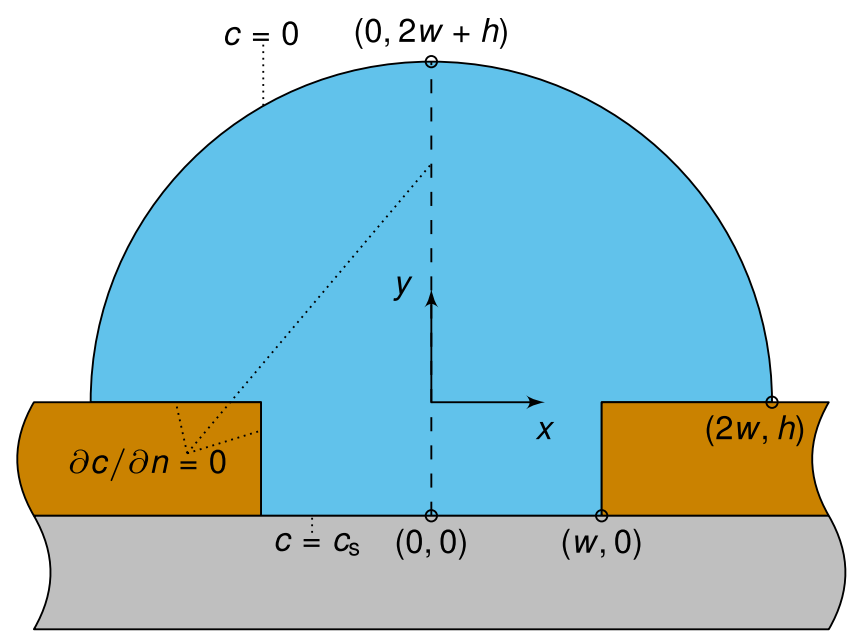

Figure 2. Schematic depiction of the calculation domain for a recessed, isolated line electrode. The boundary conditions necessary for the solution of the Laplace equation of the concentration field and the relevant geometrical dimensions are also given.

which is recessed into an insulator (Figure 2). The key dimensions to describe the geometry are the mask height $(h)$ and the mask opening half-width $(w)$. As the mask opening aspect ratio is small $(h / w<1)$ in the present case, the boundary of the diffusion layer can be described by a hemi-cylinder with radius $2 \times w$, which is centered at the coordinate $(0, h)$.

Numerical implementation.-The numerical implementation of the shape evolution simulation using a Boundary Element Method (BEM) was based on earlier work on moving boundary problems. ${ }^{38-42}$ The boundary was first discretized into line segments where the concentration was approximated along each segment using discontinuous, linear functions. ${ }^{43}$ Such boundary elements are easy to implement and allow for discontinuities at corner points and at the edge between the metal and the insulator. Subsequently, the Laplace equation was solved yielding the current density distribution along the boundary. At each time step the amount of displacement in $\mathrm{x}$ - and $\mathrm{y}$-direction of every point along the metal surface was calculated by splitting Equation 2 into its $\mathrm{x}$ - and $\mathrm{y}$-component using a finite difference approximation. ${ }^{44}$ The length of a time step was chosen small, so that the displacement in $y$-direction in the center of the evolving cavity $(x=0)$ equaled $0.005 \times w$ in order to avoid convergence problems ${ }^{39}$ and to increase accuracy. Due to symmetry, only half of the depicted solution domain was considered in order to reduce the computational effort. The metal surface and the boundary of the diffusion layer were both represented by 100 line segments each. Increasing the number of elements further lead only to negligible changes in the results. The same node density was used along the insulator and the metal surface, hence the initial boundary consisted of 570 elements in total. At each time step, node points were added and shifted along the metal and insulator boundaries to keep the node density approximately constant.

For comparison, the problem was also implemented in COMSOL Multiphysics 4.3b using a Finite Element Method (FEM). Shape changes during TMEMM were simulated with the convectiondiffusion equation and the moving mesh interface. A pre-defined etch form ${ }^{45}$ was introduced into the geometry to allow the mesh to move along the bottom surface of the insulator. This was dimensioned with a half-width of $1.005 \times w$ and an initial depth of $0.01 \times w$ and was thus small compared to the rest of the geometry.

\section{Results and Discussion}

Comparison between simulation and experiment.-The solutions to the problem of shape evolution during TMEMM using either the

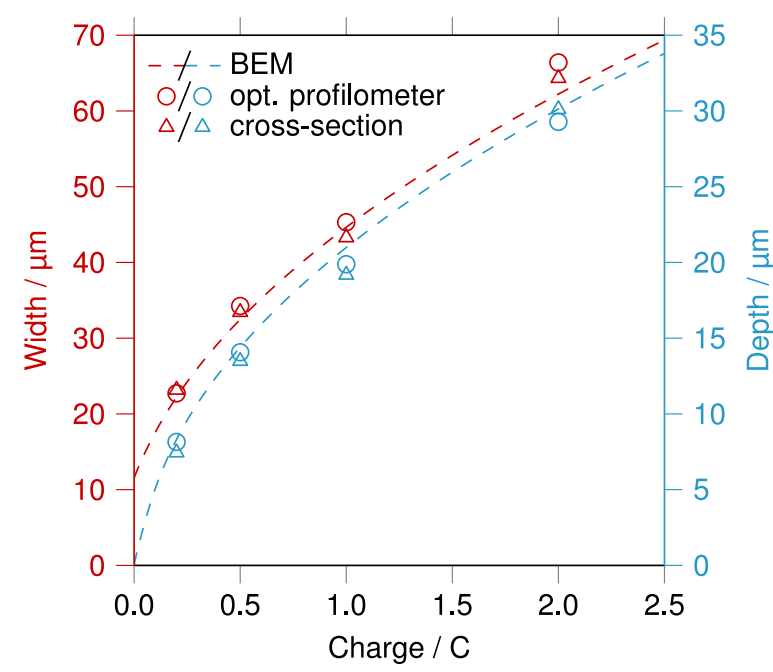

Figure 3. Dependence of the channel width and depth on the cumulative passed charge corresponding to the amount of removed material in a quiescent solution. The dashed line represents the results of BEM simulations.

BEM or FEM were generally found in excellent agreement with one another. Differences between the numerical results could be expected due to variations in the implementation of both methods in terms of initial geometry, time stepping, mesh size and remeshing. However, such variations were negligible compared to the uncertainty of the experimental results and were thus ignored. In both cases simulations were conducted using a mask height of $1.95 \mu \mathrm{m}$ and mask width of $11.5 \mu \mathrm{m}$, corresponding to a mask aspect ratio of 0.34 . Afterwards, the computed shape profiles were integrated to yield the cross-sectional area and multiplied with the sum of the center line length of each ring to obtain the removed volume and thus the cumulative passed charge. A valence of three for aluminum dissolution and $100 \%$ current efficiency were assumed in the process.

Results for the channel width and depth were obtained from optical profilometer data and from cross-sections for each ring. Both width and depth were found to increase non-linearly with the cumulative passed charge (Figure 3 ) and with time. The width and depth values of rings machined in a quiescent solution were found in good agreement with the simulation results. Under forced convection the channel dimensions increased with the distance from the axis of rotation, the variation being larger at higher rotation rates. This is explained by the dependence of the angular velocity of the RDE on radial position. When averaging the channel depth and width of samples machined at 100,400 and $900 \mathrm{rpm}$ over all rings, similar values to the ones in a quiescent solution were obtained however.

SEM and microscope images of experimental cross-sections revealed a semi-elliptical channel profile, which is characteristic for diffusion-limited material removal through a mask. ${ }^{20,38}$ One such example is shown in Figure 4. From these images experimental shape profiles were obtained via image processing and then overlaid with simulated profiles. The best agreement between the two was determined according to the overall profile shape. Comparing crosssections with respect to the position of the mask opening was not possible, because the fragile photoresist film was stripped before encasing and cross-sectioning samples. Overall the agreement with simulated profiles was good up to $100 \mathrm{rpm}$ (Figure 5), the only difference being the slightly larger width of experimental profiles toward the channel top. As rotation rate was increased to 400 and $900 \mathrm{rpm}$, the profiles tended to be asymmetric with the position of the maximum etch depth shifted radially outward. At $900 \mathrm{rpm}$ the profiles were also noticeably deeper and narrower than expected.

Based on experimental cross-sections, the etch factor $(E F)$ was calculated for each ring separately from the maximum etch depth $(d)$ and the undercut $(u)$ underneath either side of the mask opening 


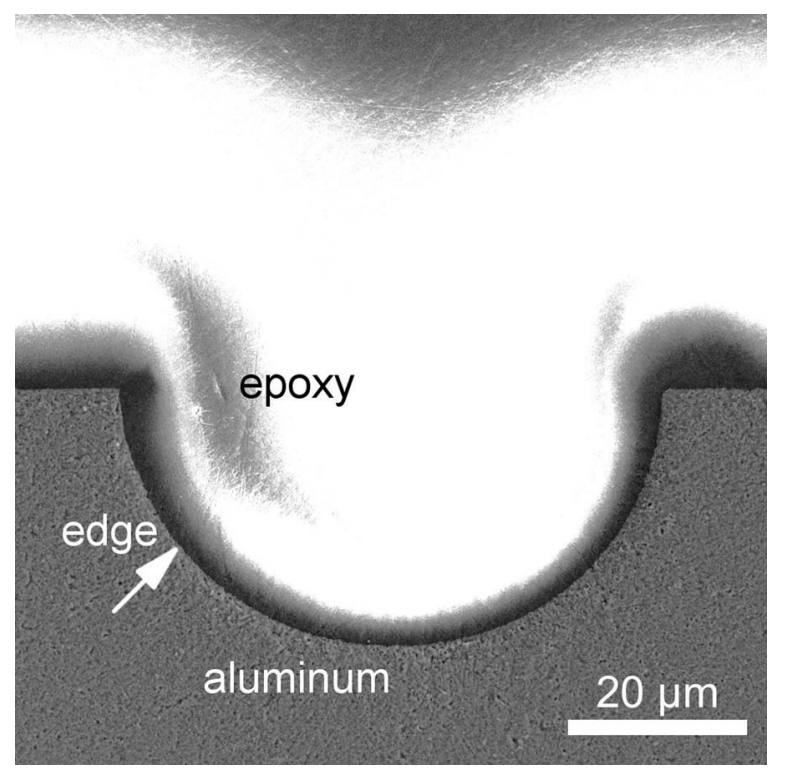

Figure 4. SEM image $(1000 \times$ magnification $)$ of the cross-section of a channel etched at an applied potential of $1.4 \mathrm{~V}$ at $100 \mathrm{rpm}$ for a cumulative charge of $2.0 \mathrm{C}$.

(a)

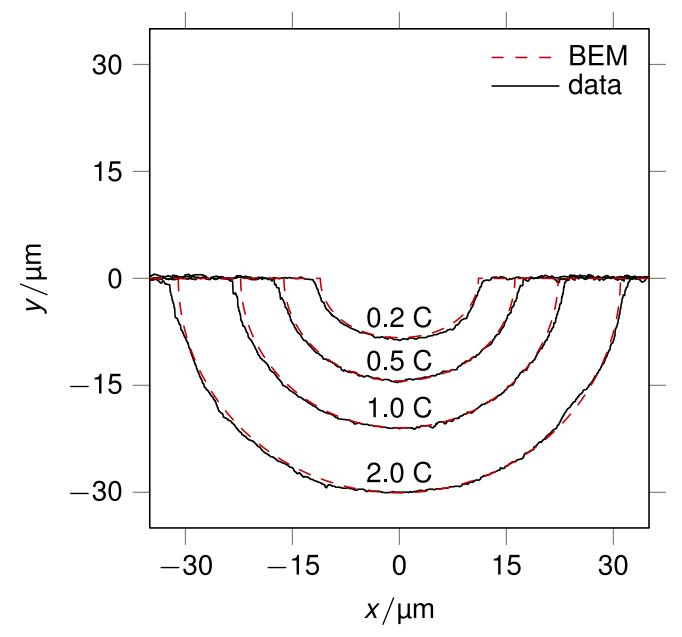

(b)

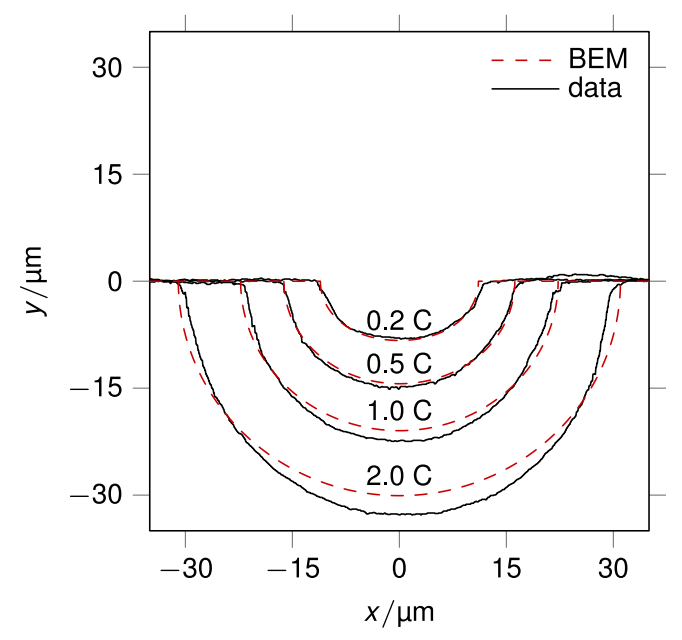

Figure 5. Comparison between simulated and experimental shape profiles for different values of the cumulative passed charge of $0.2,0.5,1.0$ and $2.0 \mathrm{C}$ at (a) $100 \mathrm{rpm}$ and (b) $900 \mathrm{rpm}$.

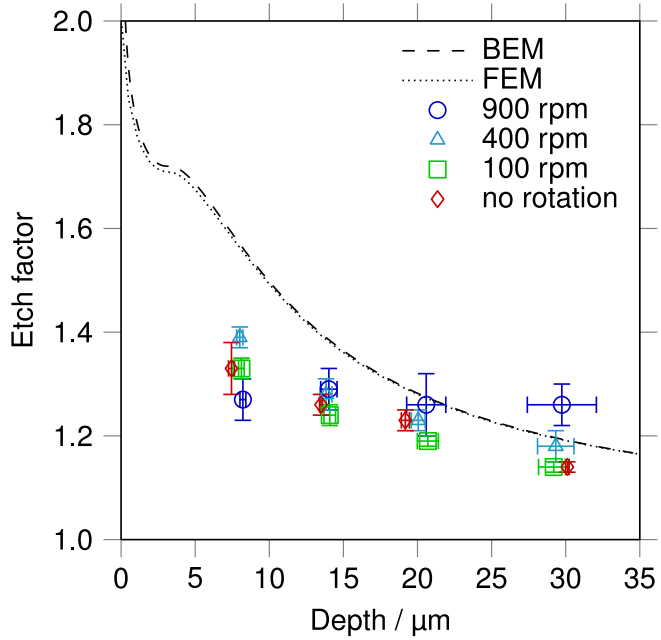

Figure 6. Relation between the etch factor and the maximum channel depth. The results are presented for different rotation rates corresponding to a quiescent solution and forced convection. Also given are the results of BEM and FEM simulations. The error bars correspond to the $95 \%$ confidence deviation.

according to the following formula:

$$
E F=d / u
$$

For a given experiment no influence of radial position was detected even at high rotation rates, hence the results are reported as average values. This is because the difference in the amount of removed material between the five rings was small compared to the total amount. In a quiescent solution and under the conditions of forced convection up to $400 \mathrm{rpm}$ the etch factor was found to decrease with increases in channel depth and ranged between 1.4 and 1.1 (Figure 6). Up to $400 \mathrm{rpm}$ the experimental results agreed with each other, but deviated from the simulated etch factor, the difference being larger for shallower channels. In contrast, the etch factor stayed roughly constant just below 1.3 at $900 \mathrm{rpm}$.

The deviations in terms of channel width and etch factor even in the absence of convection can be explained based on SEM images of machined channels (Figure 7). On either side of each channel, where the channel wall met the mechanically polished surface, a small 0.5 to $2 \mu \mathrm{m}$ wide region with a surface structure similar to the machined channel surface was frequently found. On cross-sections this region was not detectable, thus only little material was removed in this place. The simulation results predicted a $90^{\circ}$ angle at the intersection between the channel wall and the bottom surface of the mask. In practice, the channel wall followed a wedge-like shape in the vicinity of the mask exposing a larger surface area than expected to the electrolyte. From previous work it is known, that aluminum oxide continuously forms and dissolves during mass-transfer limited, electrochemical dissolution in phosphoric acid. ${ }^{37}$ In the present work, the disk samples were handled in the presence of air, hence a thin air-formed alumina film is presumed to cover the surface underneath the photoresist mask. We hypothesize that this air-formed film may be dissolved preferentially leading to more undercutting of the mask. Alternatively, problems with photoresist adhesion may cause a larger part of the surface to be exposed to the electrolyte.

The machined rings were also characterized in terms of their surface roughness. The average surface roughness $\left(R_{\mathrm{a}}\right)$ and root mean squared roughness $\left(R_{\mathrm{q}}\right)$ values were determined from line scans taken from optical profilometer data along the mechanically polished surface and the bottom of machined channels. Both quantities and their standard deviation were computed for the ring with the largest diameter only. For the initial mechanically polished surface these values were determined as $R_{\mathrm{a}}=14 \pm 4 \mathrm{~nm}$ and $R_{\mathrm{q}}=18 \pm 6 \mathrm{~nm}$. After shape evolution experiments these figures increased slightly to $R_{\mathrm{a}}=28 \pm 6 \mathrm{~nm}$ and $R_{\mathrm{q}}=40 \pm 9 \mathrm{~nm}$, where aluminum had been electrochemically 

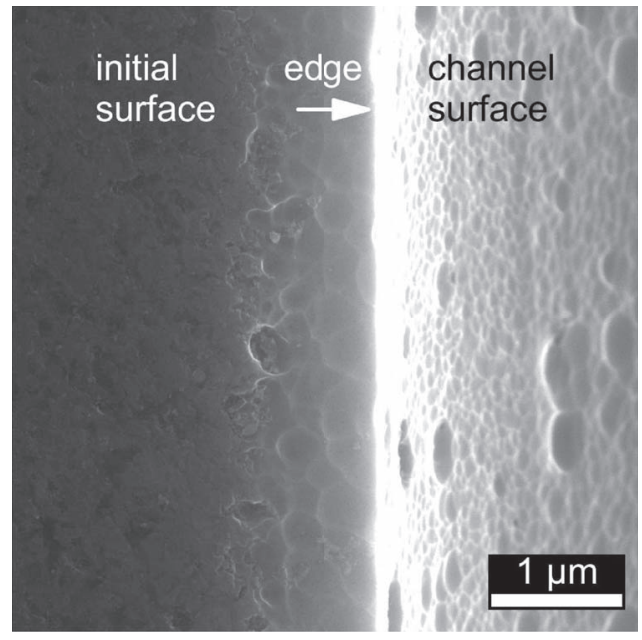

alumina

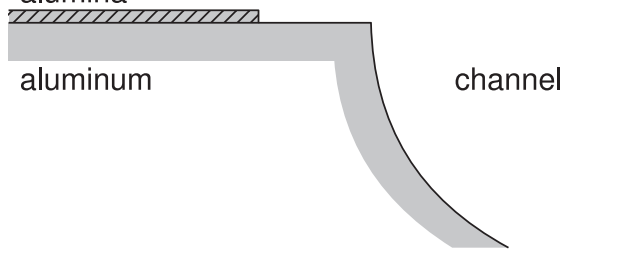

Figure 7. SEM image $(20000 \times$ magnification) of the border region between the mechanically polished initial surface and the machined channel surface. The arrow marks the position where the vertical channel side wall meets the horizontal plateau. Also shown is a schematic cross-section indicating partial removal of the air-formed oxide film in the vicinity of the channel edge.

dissolved. Similar results have been obtained after electropolishing ${ }^{46}$ and electrochemical micromachining ${ }^{27}$ of aluminum in viscous electrolytes. Surface roughness values determined after TMEMM of aluminum in a mixture of acetic and perchloric acid $^{28}$ are about one order of magnitude higher compared to the present work. No influence of rotation rate or amount of removed material was found.

Influence offorced convection.-On an aluminum RDE the magnitude of the mass-transfer limited current depends directly on the square root of rotation rate. ${ }^{17,37}$ Likewise, the form of current transients measured during shape evolution experiments at an applied potential of $1.4 \mathrm{~V}$ depended on the conditions of mass transfer (Figure 8). In a quiescent solution the current quickly attained a peak value and

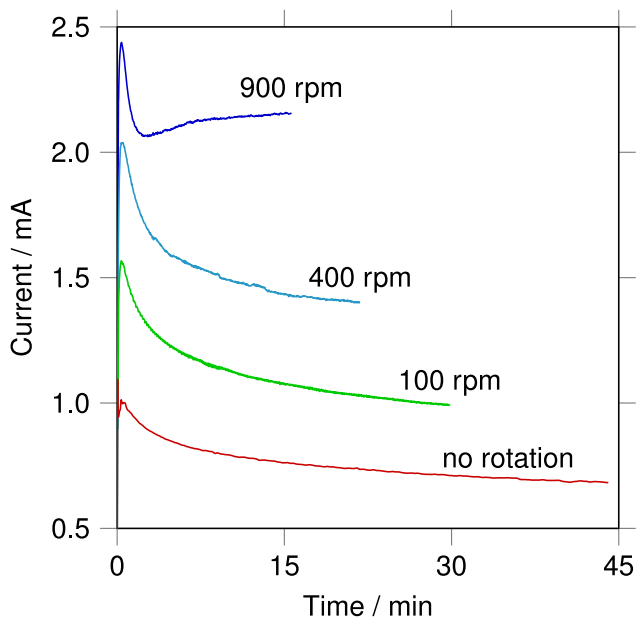

Figure 8. Current transients measured at an applied potential of $1.4 \mathrm{~V}$ and different rotation rates for a cumulative passed charge of $2.0 \mathrm{C}$.

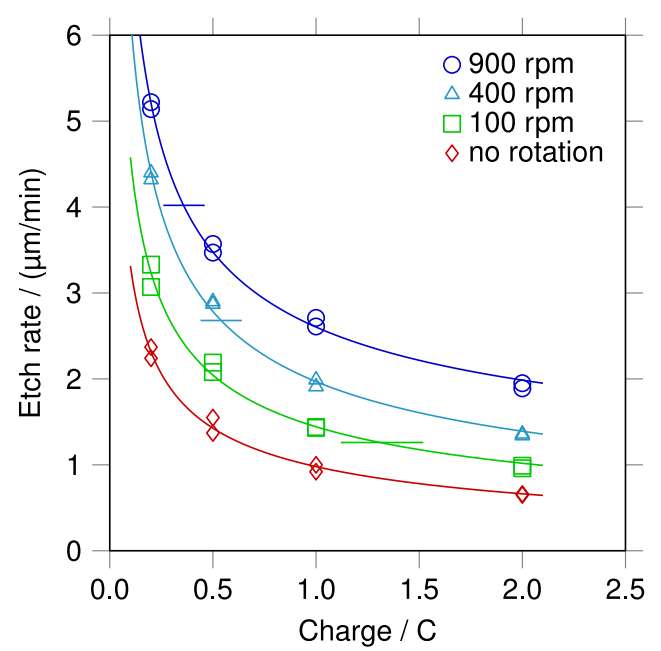

Figure 9. Variation of the etch rate for different amounts of the cumulative passed charge. The solid lines were added for clarity and represent a linear regression fit assuming the etch rate is proportional to the inverse square root of the cumulative passed charge. The horizontal lines indicate the etch rate for a mask opening of infinite size at the respective rotation rate.

then continuously decreased over time. This behavior is known from TMEMM of titanium in a sulfuric acid-methanol electrolyte ${ }^{38}$ and arises from the establishment and subsequent leveling of concentration gradients along the metal surface as the etch depth increases. Increasing rotation rate to 100 and $400 \mathrm{rpm}$ lead to similarly shaped current transients. At $900 \mathrm{rpm}$ however, the current quickly reached a plateau and stayed constant. This indicated a change in the relative importance of diffusive and convective mass transfer during the dissolution process as rotation rate increases.

Likewise, the etch rate - here defined as the ratio of maximum etch depth to etch time - was found to depend on the conditions of mass transfer (Figure 9). In the absence of convection the etch rate ranged between 0.6 and $2.4 \mu \mathrm{m} / \mathrm{min}$, which is typical for TMEMM of aluminum in electrolytes consisting of viscous mineral acids. ${ }^{27,28}$ At $900 \mathrm{rpm}$ etch rates ranging between 1.9 and $5.3 \mu \mathrm{m} / \mathrm{min}$ were calculated. In all cases the etch rate decreased sharply with the cumulative passed charge. This is explained by the aforementioned decrease in the total current over time, a decline in the average current density as the channel surface grows and an increase in the amount of lateral to vertical material removal as the channels deepen. The shift from purely diffusive to mixed convective-diffusive mass transfer was determined from a double logarithmic plot of etch depth and etch rate against time (Figure 10), respectively. For a purely diffusion-limited etch process the maximum etch depth is proportional to the square root of time $e^{20,21}$ corresponding to a slope of 0.5 in a log-log plot. Similarly, the etch rate depends on the inverse square root of time in this case yielding a slope of -0.5 . In the absence of convection the slopes computed via linear regression agreed favorably with the theoretical values. As rotation rate increased the slopes deviated more and more from these values indicating the growing influence of convective mass transfer.

It should be noted that lower etch rates must be expected when etching more tightly-spaced patterns consisting of larger features. It is generally understood that the etch rate depends strongly on the geometry, size and spacing of the mask openings within a pattern. ${ }^{47}$ For comparison with the above results we have estimated the etch rate for a mask opening of infinite size from limiting current data measured on an aluminum $\operatorname{RDE}^{37}$ via Faraday's law at $100 \%$ current efficiency $\left(M=26.98 \mathrm{~g} / \mathrm{mol}, \rho=2.70 \mathrm{~g} / \mathrm{cm}^{3}, z=3\right)$. The results at 100,400 and $900 \mathrm{rpm}$ for this theoretical etch rate correspond to the horizontal lines in Figure 9. It was found, that etching through a mask opening of finite size proceeded faster for shallow channels. In contrast, deep channels etched at a lower rate compared to the theoretical value. This observation coincides with theoretical results and interpretations 
(a)

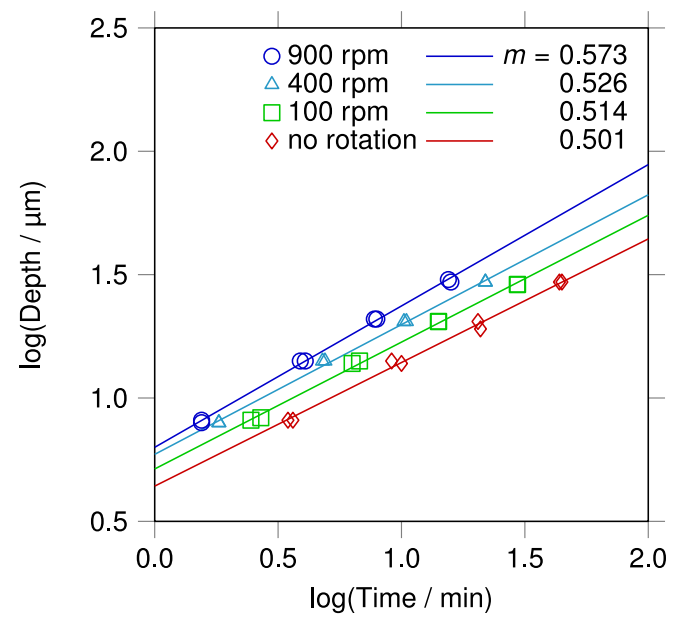

(b)

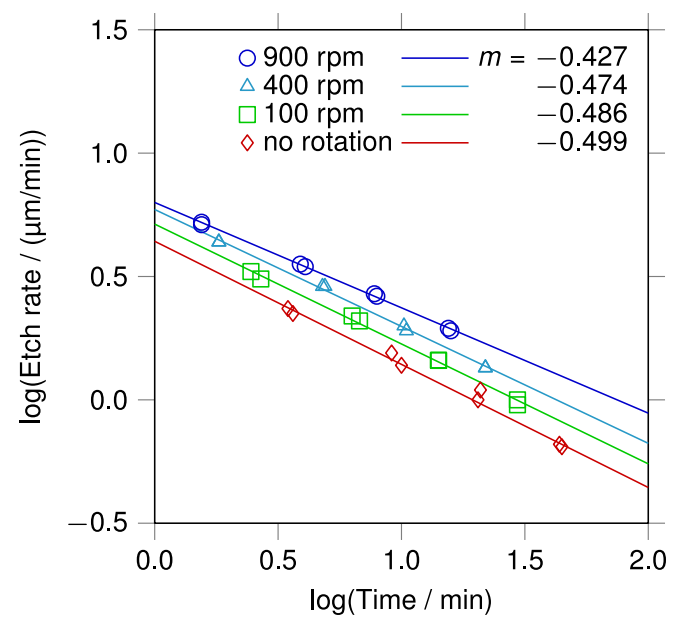

Figure 10. Double logarithmic plot of (a) maximum etch depth and (b) etch rate against etch time. Also given is the slope of a linear regression fit under the respective hydrodynamic conditions.

thereof obtained by Kuiken ${ }^{26}$ for purely diffusion-limited, chemical etching. During the early stages of etching a decrease in the mask opening size leads to steeper concentration gradients in the vicinity of the opening. At later times however, smaller mask openings also limit the flux of material more severely.

As mentioned above, increasing rotation rate also lead to changes in the overall shape profile of machined channels. In addition to the changes in depth and width, a shift in the position of the left and right channel wall was observed. In a quiescent solution and when etching at $100 \mathrm{rpm}$, material removal occurred at equal rates to the left and right side of the mask opening. The profile of these channels was thus centered on the middle of the mask opening. In contrast, microscope images of channels etched at 400 and $900 \mathrm{rpm}$ showed uneven undercutting between both sides of the mask opening (Figure 11). In general, more undercutting occurred underneath the part of the mask, which lay further away from the axis of rotation. The difference in undercutting increased with radial position at constant rotation rate and with rotation rate at constant radial position. These observations

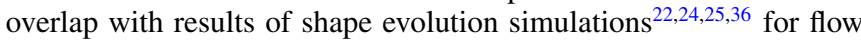
past a partially covered trench, namely that under forced convection and in the presence of a thin mask more undercutting occurs along the downstream edge of the trench. Additionally, the difference in undercutting depends on the velocity in the vicinity of the mask opening. In the present case this corresponds to radial flow past a recessed ring.

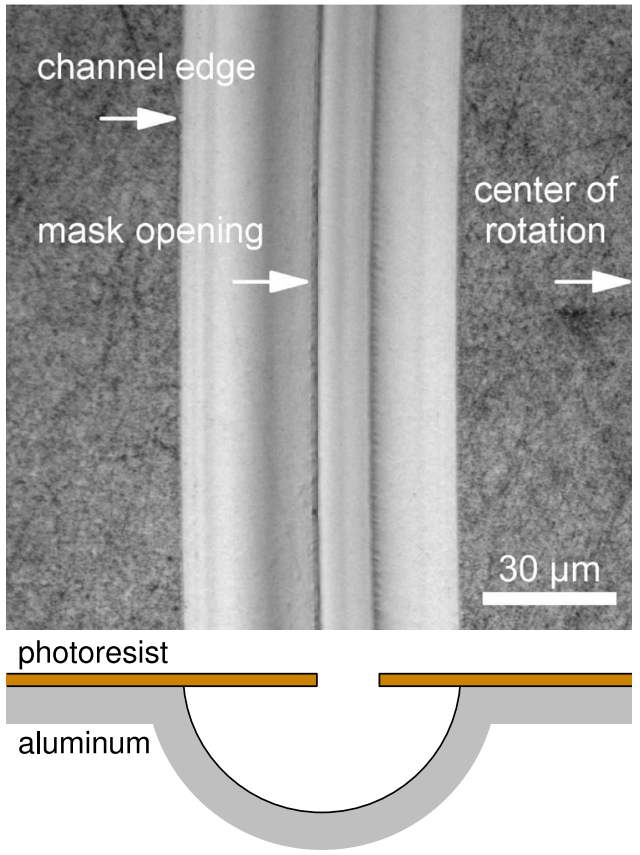

Figure 11. Microscope image (50× magnification) of a channel machined at an applied potential of $1.4 \mathrm{~V}$ at $900 \mathrm{rpm}$ for a cumulative passed charge of $2.0 \mathrm{C}$. The center of rotation lies to the right outside of this image. The schematic cross-section highlights the displacement of the channel profile relative to the mask opening.

Typically, the relative importance of convective and diffusive mass transfer is expressed in the form of the Péclet number $(P e=u L / D)$ using a characteristic velocity $(u)$ and length scale $(L)$ and the diffusion coefficient $(D)$. In studies on cavity etching through an insulating mask in the presence of convection, ${ }^{22-24,36}$ the mask opening half-width is used as the characteristic length, hence $L$ equaled $5.75 \mu \mathrm{m}$. Data on the diffusion coefficient of $\mathrm{Al}^{3+}$ in phosphoric acid is unavailable to our knowledge. Instead, we followed the reasoning of Datta et al. ${ }^{48}$ and assumed, that the diffusion coefficient of metal ions in concentrated electrolytes depends mainly on viscosity and electrolyte temperature and not on the type of metal ion. Thus, we used a least squares fit of diffusion coefficient and viscosity data determined for $\mathrm{Cu}^{2+}$ in phosphoric acid at $25^{\circ} \mathrm{C}$ to estimate $D$ : $D=5.7 \times 10^{-10} \nu^{-1.06}\left(\mathrm{~m}^{2} / \mathrm{s}\right){ }^{49}$ Using density $\left(\rho=1685 \mathrm{~kg} / \mathrm{m}^{3}\right)^{50}$ and viscosity data $\left(\eta=39.3 \times 10^{-3} \mathrm{~Pa} \mathrm{~s}, \nu=2.33 \times 10^{-5} \mathrm{~m}^{2} / \mathrm{s}\right)^{51}$ of $85 \%$ phosphoric acid yielded $D=2.0 \times 10^{-11} \mathrm{~m}^{2} / \mathrm{s}$ at $25^{\circ} \mathrm{C}$. This value was subsequently corrected for viscosity and temperature changes via the Stokes-Einstein equation resulting in $D=9.5 \times 10^{-11} \mathrm{~m}^{2} / \mathrm{s}$ at $75^{\circ} \mathrm{C}$. Finally, we estimated the characteristic velocity in the vicinity of the mask opening from the velocity gradient and the characteristic length via $u \sim \nabla u L .{ }^{26}$ Due to axial symmetry and the proximity to the surface only the radial velocity component $\left(u_{r}\right)$ was of interest, which for a RDE is given by $u_{r}=0.51 \times r \omega^{3 / 2} v^{-1 / 2} y^{52}$ close to the surface. Likewise, only the derivative with respect to the longitudinal coordinate $(y)$ was considered, resulting in the following equation for the characteristic velocity $u=0.51 \times r \omega^{3 / 2} \nu^{-1 / 2} L$. It was thus possible to estimate the Péclet number at each rotation rate and for each of the five rings separately.

In order to identify a range of Péclet numbers, for which mass transfer shifts from diffusion to mixed convection-diffusion control, the radial displacement of each ring was plotted over the Péclet number (Figure 12). The data was limited to samples machined for a cumulative passed charge of $2.0 \mathrm{C}$, where the displacement was most noticeable. For $P e \leq 10$ no preferential undercutting on either side was observed and the channels remained centered relative to the mask opening. This coincided with the observation, that experimental results obtained at $100 \mathrm{rpm}$ still agreed well with the model based on 


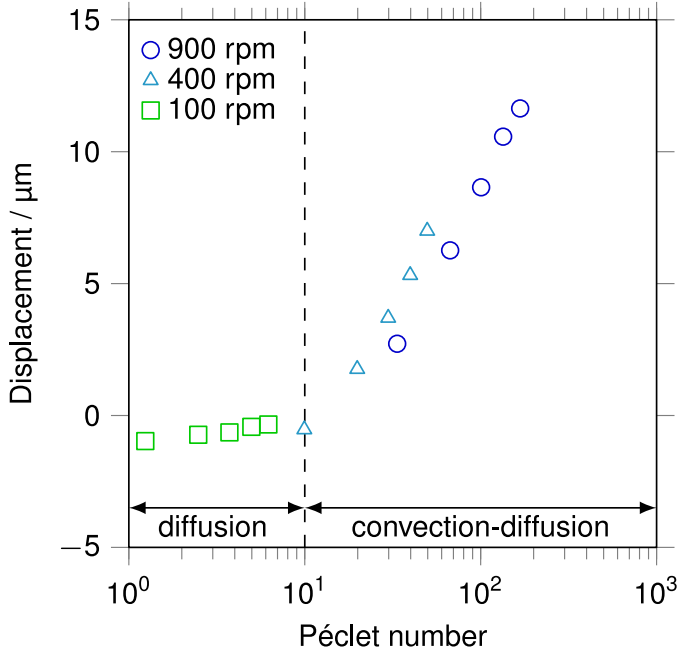

Figure 12. Variation of the radial displacement of rings machined into the surface of an aluminum RDE depending on the Péclet number in the vicinity of the respective mask opening. The Péclet number range is indicated, for which the shape evolution model based on diffusive mass transfer is in good agreement with experimental results.

diffusive mass transfer. For $P e \geq 20$ however, the radial displacement became noticeable and increased approximately linearly with the logarithm of the Péclet number. We conjecture, that these results are transferable to other flow conditions for example to TMEMM of aluminum in a flow channel or impinging jet cell. This would enable estimating an electrolyte flow rate and hence velocity for which the displacement of machined channels in the direction of flow is nonexistent or negligible. The above results show, that a RDE itself is not suitable for TMEMM at higher Péclet numbers due to the radial dependence of the etch rate and channel displacement. It should be noted however, that it enables one to test for the influence of flow conditions over a wide range of Péclet numbers. Only a few experiments are necessary to that end.

Influence of potential.-Mass-transfer limited anodic dissolution of aluminum in concentrated phosphoric acid at elevated temperatures is characterized by a limiting current plateau, that starts at about -0.2 to $0.3 \mathrm{~V}$ vs. $\mathrm{Ag} \mid \mathrm{AgCl}$ and extends over several volts. ${ }^{17,37}$ Thus, a wide potential range is in principle accessible for TMEMM of aluminum. In practice, current transients measured during TMEMM differ depending on the applied potential along the limiting current plateau (Figure 13). At $1.4 \mathrm{~V}$ the current decreases steadily over time, whereas it fluctuates after a few minutes at an applied potential of $5.4 \mathrm{~V}$. A larger decrease from the peak current value precedes these fluctuations. Both observations indicate the gradual formation of gas bubbles along the metal surface, which subsequently detach at periodic intervals. These bubbles are not observable themselves due to their small size, but their presence is evident from SEM images of machined channels (inserts in Figure 13). At $5.4 \mathrm{~V}$ the channel shape is non-uniform and the channel width and depth vary considerably. This is due to partial or complete blockage of the openings in the photoresist film with gaseous reaction products leading to locally lower rates of material removal. The most likely cause is oxygen evolution. This is backed up by earlier reports on mass-transfer limited anodic dissolution of aluminum in phosphoric acid, namely the observation that gas evolves at sufficiently high potentials ${ }^{53}$ and that the apparent valence of aluminum dissolution in phosphoric acid is slightly lower than three, ${ }^{17}$ corresponding to a current efficiency of less than $100 \%$. It should be stressed, that a small percentage of the current going toward oxygen evolution is sufficient to explain the observed effects due to the large molar volume of gaseous oxygen.

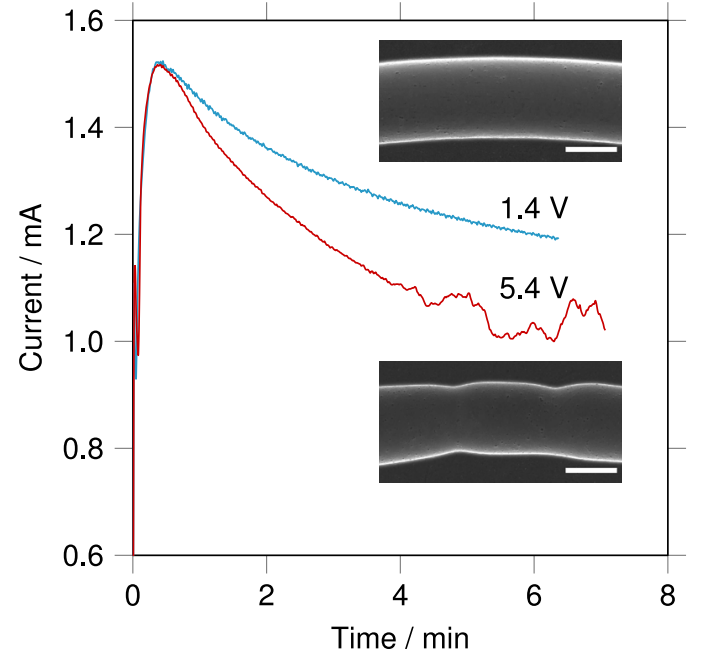

Figure 13. Current transients measured at a rotation rate of $100 \mathrm{rpm}$ at two different applied potentials along the limiting current plateau for a cumulative passed charge of $0.5 \mathrm{C}$. The inserts are SEM images $(1000 \times$ magnification) depicting channels machined at the respective conditions. The scale bar corresponds to $20 \mu \mathrm{m}$ in each case.

Microfluidic example structures.-To investigate the feasibility of TMEMM of aluminum for the fabrication of microfluidic devices, several test patterns were machined into the surface of disk samples. Based on previous experience, electrochemical dissolution was conducted at an applied potential of $1.4 \mathrm{~V}$ in a quiescent solution. The amount of removed material was controlled by measuring the cumulative passed charge. One such pattern represents a micro-mixer structure based on a meandering channel design ${ }^{54}$ (Figure 14). The structure consists of two inlet chambers, which lead into a respective inlet channel. Both inlet channels merge in a mixing section followed by a single meandering channel and an outlet chamber. The mask opening was $50 \mu \mathrm{m}$ wide for each inlet channel and $100 \mu \mathrm{m}$ wide for the mixing channel. The inlet and outlet chambers were designed with a diameter of $1 \mathrm{~mm}$. After TMEMM the width of the inlet and the mixing channel was 95 and $145 \mu \mathrm{m}$, respectively. Optical profilometer data showed that the channels reached similar depths of 30 and $35 \mu \mathrm{m}$. SEM images revealed a smooth and even surface finish and well-defined channel walls. The inlet and outlet chamber showed large variations in etch depth however. Along the edge of each chamber ran a trench about $20 \mu \mathrm{m}$ deeper than the chamber center. A similar variation in etch depth occurred when machining arrays of parallel channels, which may be used within a micro-reactor or micro-heat exchanger. In this case the outermost channel of the pattern showed the highest etch rate, leading to a larger etch depth compared to the rest of the pattern.

The problem is known from experimental and theoretical studies $^{3,42,55}$ and arises due to differences in the shape of the diffusion layer boundary. ${ }^{47}$ Over large mask openings or dense patterns of small mask openings the diffusion layer boundary is parallel to the surface and mass transfer occurs in one dimension only. In contrast, the diffusion layer attains a curved shape at the edge of mask openings or patterns, leading to enhanced mass transfer in this region. Several solutions to the problem have been proposed, some of which are applicable to TMEMM under mass-transfer limited conditions. Concentration gradients along the edge of a pattern of mask openings can be leveled by introducing so-called current robber areas surrounding the pattern. ${ }^{3}$ Also, large mask openings can be divided into a number of smaller mask openings, a large chamber thus being formed by the merging of many smaller cavities. ${ }^{42,55}$ In theory reducing the thickness of the diffusion layer via forced convection should also reduce the dependence of the etch rate on size effects. ${ }^{47}$ In future work these 


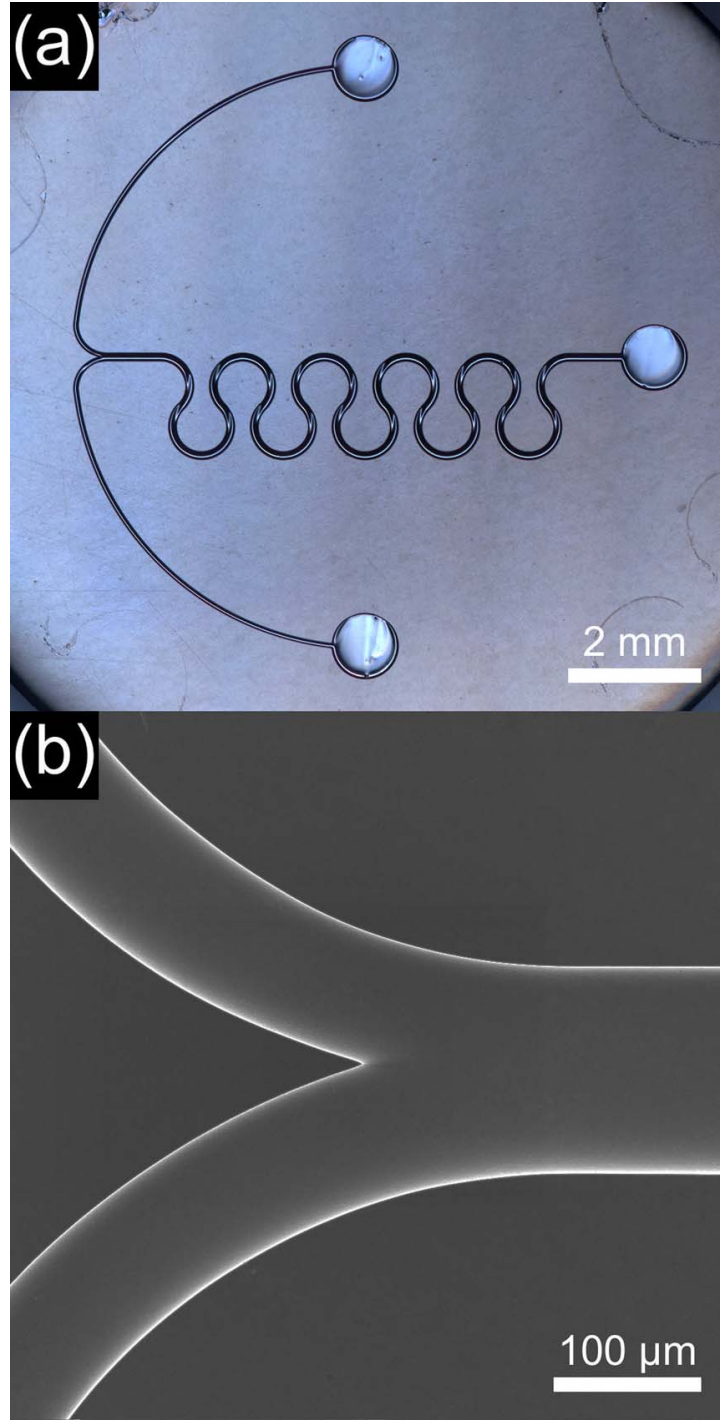

Figure 14. (a) Micromixer consisting of two inlet channels leading into a serpentine mixing channel. The micromixer was machined into the surface of an aluminum disk $15 \mathrm{~mm}$ in diameter at an applied potential of $1.4 \mathrm{~V}$ at $75^{\circ} \mathrm{C}$ in a quiescent solution for a cumulative passed charge of $10 \mathrm{C}$. (b) SEM image (200× magnification) showing where the inlet channels merge into the mixing channel. The two inlets are $90 \mu \mathrm{m}$ wide and $45 \mu \mathrm{m}$ deep while the mixing channel is $140 \mu \mathrm{m}$ wide and $50 \mu \mathrm{m}$ deep.

options will be explored for TMEMM of aluminum in order to yield more uniform etch rates over the whole of a pattern of mask openings.

\section{Conclusions}

In this work, aluminum disks were electrochemically dissolved through photoresist masks in $85 \%$ phosphoric acid at $75^{\circ} \mathrm{C}$ using a custom-made RDE setup. The influence of applied potential, cumulative passed charge and hydrodynamic conditions on the dimensions, shape profile and uniformity of resulting micro-channels was studied and compared with a shape evolution model based on diffusive mass transfer. ${ }^{38}$ The channels and cross-sections made thereof were characterized via SEM, light microscopy and optical profilometry. The experimental and model results agreed well with each other in terms of the ratio of vertical to lateral material removal, the resulting channel dimensions and the overall shape profile up to a rotation rate of $100 \mathrm{rpm}$. At higher rotation rates deviations between experiment and simulation indicated a shift from purely diffusive to mixed convective-diffusive mass transfer. This shift was characterized by estimating the Péclet number in the vicinity of each mask opening and a range of Péclet numbers were identified, for which the shape evolution process is described well by the model. In addition, preliminary results on the machining of complex microfluidic structures demonstrate that TMEMM of aluminum is a feasible alternative to other micromachining techniques.

\section{Acknowledgments}

The authors thank Graham Mitchell for the manufacture of aluminum disks and technical support, Roger Reeves and Cather Simpson for access to the polishing machine and the optical profilometer, respectively, Helen Devereux and Gary Turner for technical support in the Nanolab, Mathieu Sellier for helpful discussions regarding COMSOL and Mike Flaws for support with SEM imaging.

\section{References}

1. M. Datta and D. Landolt, Electrochim. Acta, 45, 2535 (2000).

2. D. Landolt, P.-F. Chauvy, and O. Zinger, Electrochim. Acta, 48, 3185 (2003).

3. D. M. Allen, PCMI J., 27, 10 (1987).

4. M. Datta and D. Harris, Electrochim. Acta, 42, 3007 (1997).

5. M. Datta, IBM J. Res. Dev., 42, 655 (1998).

6. K. Crämer, F. Fürstenau, and H. Meyer, in Proceedings / MICRO.tec 2000 : ap plications, trends, visions / VDE World Microtechnologies Congress (Expo 2000), Hannover, Germany (2000).

7. Z. Anxionnaz, M. Cabassud, C. Gourdon, and P. Tochon, Chem. Eng. Process., 47, 2029 (2008)

8. G. Kolb, Chem. Eng. Process., 65, 1 (2013).

9. G. Scotti, P. Kanninen, T. Kallio, and S. Franssila, J. Microelectromech. S., 23, 372 (2014).

10. K. E. Mattsson, Microelectron. Eng., 19, 199 (1992).

11. D. M. Allen, in Proceedings 2nd International \& 23rd AIMTDR Conference, Indian Institute of Technology Madras, Chennai, India (2008).

12. G. E. Thompson, Thin Solid Films, 297, 192 (1997).

13. W. Lee and S.-J. Park, Chem. Rev, 114, 7487 (2014).

14. G. Wießmeier and D. Hönicke, J. Micromech. Microeng., 6, 285 (1996).

15. E. V. Rebrov, M. H. J. M. de Croon, and J. C. Schouten, Catal. Today, 69, 183 (2001)

16. J. C. Ganley, E. G. Seebauer, and R. I. Masel, AIChE J., 50, 829 (2004).

17. R. Vidal and A. C. West, J. Electrochem. Soc., 145, 4067 (1998).

18. D. Landolt, Electrochim. Acta, 32, 1 (1987).

19. D. Landolt, in Proceedings 5th International Symposium on Electrochemical Ma chining Technology INSECT 2009, Fraunhofer Institute for Ceramic Technologies and Systems, Dresden, Germany (2009).

20. H. K. Kuiken, J. J. Kelly, and P. H. L. Notten, J. Electrochem. Soc., 133, 1217 (1986).

21. P. H. L. Notten, J. J. Kelly, and H. K. Kuiken, J. Electrochem. Soc., 133, 1226 (1986).

22. C. B. Shin and D. J. Economou, J. Electrochem. Soc., 136, 1997 (1989).

23. C. B. Shin and D. J. Economou, Int. J. Heat Mass Tran., 33, 2191 (1990).

24. C. B. Shin and D. J. Economou, J. Electrochem. Soc., 138, 527 (1991).

25. C. H. Driesen, Ph. D. thesis, University of Twente, 1999

26. H. K. Kuiken, J. Eng. Math., 45, 75 (2003).

27. Y. Kim, S. Youn, Y.-H. Cho, H. Park, B. G. Chang, and Y. S. Oh, J. Micromech Microeng., 21, 015019 (2011).

28. T. Kikuchi, Y. Wachi, M. Sakairi, and R. O. Suzuki, Microelectron. Engn., 111, 14 (2013).

29. T. Kikuchi, Y. Wachi, T.-A. Takahashi, M. Sakairi, and R. O. Suzuki, Electrochim. Acta, 94, 269 (2013).

30. S. Qian and F. Ji, in Proceedings AASRI International Conference on Industrial Electronics and Applications (IEA 2015), London, UK (2015).

31. E. Rosset and D. Landolt, Precis. Eng., 11, 79 (1989).

32. E. Rosset, M. Datta, and D. Landolt, J. Appl. Electrochem., 20, 69 (1990).

33. C. Winkelmann and W. Lang, Int. J. Mach. Tool. Manu., 72, 25 (2013).

34. R. P. Tijburg, J. G. M. Ligthart, H. K. Kuiken, and J. J. Kelly, J. Electrochem. Soc., 150, C440 (2003)

35. J. J. Sudirham, R. M. J. van Damme, and J. J. W. van der Vegt, in Proceedings European Congress on Computational Methods in Applied Sciences and Engineering ECCOMAS 2004, Jyväskylä, Finland (2004).

36. P. Rath and J. C. Chai, Numer. Heat Tr. B-Fund., 53, 143 (2007).

37. T. Baldhoff and A. T. Marshall, J. Electrochem. Soc., 164, C46 (2017).

38. C. Madore, O. Piotrowski, and D. Landolt, J. Electrochem. Soc., 146, 2526 (1999).

39. A. C. West, C. Madore, M. Matlosz, and D. Landolt, J. Electrochem. Soc., 139, 499 (1992).

40. G.-J. Kwon, H.-Y. Sun, and H.-J. Sohn, J. Electrochem. Soc., 142, 3016 (1995).

41. R. V. Shenoy and M. Datta, J. Electrochem. Soc., 143, 544 (1996).

42. R. V. Shenoy, M. Datta, and L. T. Romankiw, J. Electrochem. Soc., 143, 2305 (1996).

43. W.-T. Ang, A Beginner's Course in Boundary Element Methods, Universal Publishers, Boca Raton (2007).

44. C. Clerc and D. Landolt, Electrochim. Acta, 29, 787 (1984).

45. A. Ivanov and U. Mescheder, in Proceedings COMSOL Conference 2015, Grenoble, France (2015) 
46. D. Ma, S. Li, and C. Liang, Corros. Sci., 51, 713 (2009).

47. M. Köhler, Etching in Microsystem Technology, Wiley-VCH, Weinheim (1999).

48. M. Datta, L. F. Vega, L. T. Romankiw, and P. Duby, Electrochim. Acta, 37, 2469 (1992).

49. S. H. Glarum and J. H. Marshall, J. Electrochem. Soc., 132, 2872 (1985).

50. E. P. Egan and B. B. Luff, Ind. Eng. Chem., 47, 1280 (1955).
51. D.-T. Chin and H. H. Chang, J. Appl. Electrochem., 19, 95 (1989).

52. V. G. Levich, Physicochemical hydrodynamics, Prentice-Hall, Englewood Cliffs (1962).

53. M. Zamin, P. Mayer, and M. K. Murthy, J. Electrochem. Soc., 123, 1377 (1976).

54. F. Schönfeld and S. Hardt, AIChE J., 50, 771 (2004).

55. T. Mineta, Sensor. Actuat. A-Phys., 114, 536 (2004). 